Neurology

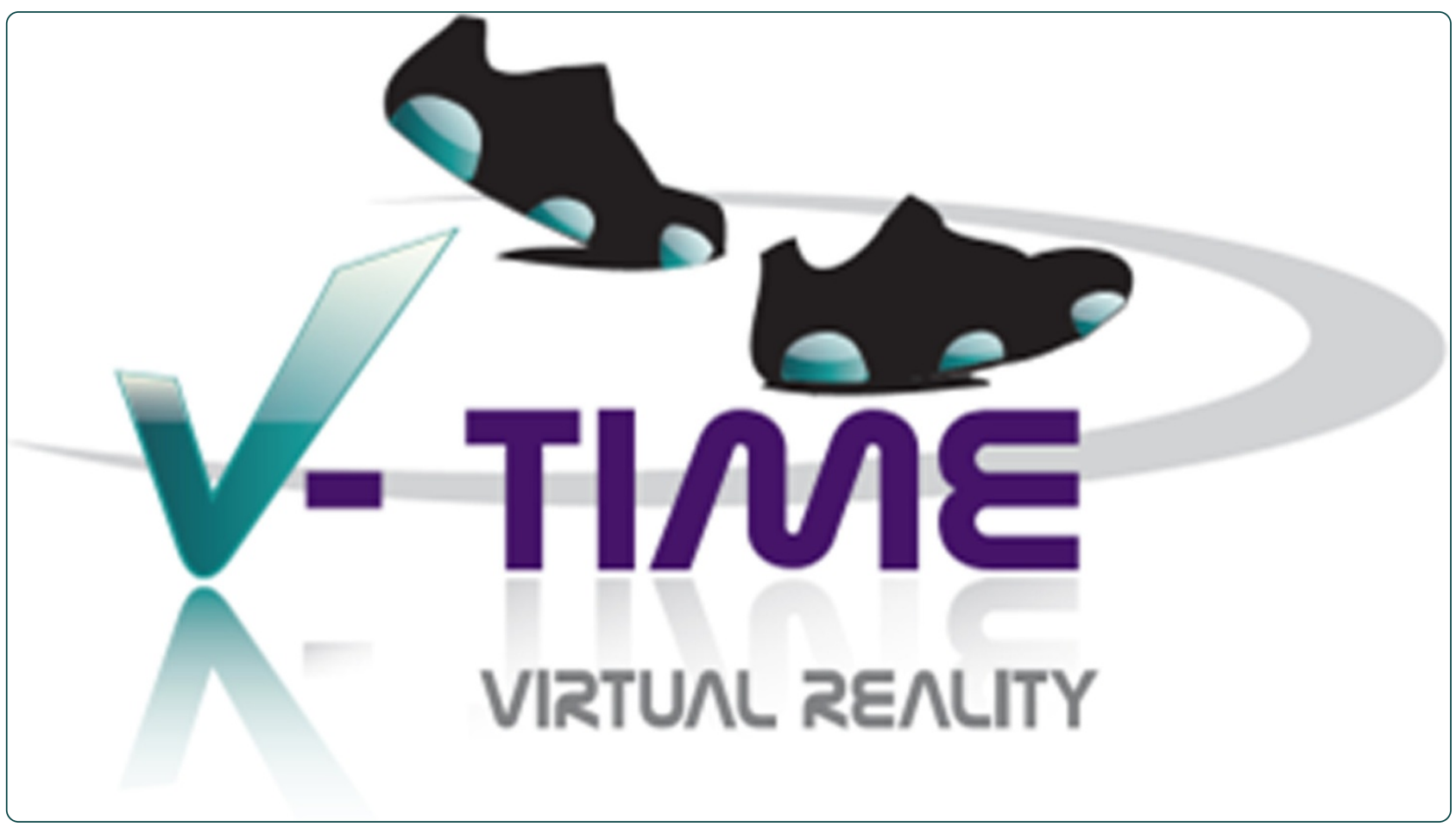

V-TIME: a treadmill training program augmented by virtual reality to decrease fall risk in older adults: study design of a randomized controlled trial

Mirelman et al. 


\title{
V-TIME: a treadmill training program augmented by virtual reality to decrease fall risk in older adults: study design of a randomized controlled trial
}

Anat Mirelman 1,2,8*, Lynn Rochester ${ }^{3}$, Miriam Reelick ${ }^{4}$, Freek Nieuwhof ${ }^{4}$, Elisa Pelosin ${ }^{5}$, Giovanni Abbruzzese ${ }^{5}$, Kim Dockx ${ }^{6}$, Alice Nieuwboer ${ }^{6}$ and Jeffrey M Hausdorff ${ }^{1,7,8}$

\begin{abstract}
Background: Recent work has demonstrated that fall risk can be attributed to cognitive as well as motor deficits. Indeed, everyday walking in complex environments utilizes executive function, dual tasking, planning and scanning, all while walking forward. Pilot studies suggest that a multi-modal intervention that combines treadmill training to target motor function and a virtual reality obstacle course to address the cognitive components of fall risk may be used to successfully address the motor-cognitive interactions that are fundamental for fall risk reduction. The proposed randomized controlled trial will evaluate the effects of treadmill training augmented with virtual reality on fall risk.

Methods/Design: Three hundred older adults with a history of falls will be recruited to participate in this study. This will include older adults ( $n=100)$, patients with mild cognitive impairment $(n=100)$, and patients with Parkinson's disease $(n=100)$. These three sub-groups will be recruited in order to evaluate the effects of the intervention in people with a range of motor and cognitive deficits. Subjects will be randomly assigned to the intervention group (treadmill training with virtual reality) or to the active-control group (treadmill training without virtual reality). Each person will participate in a training program set in an outpatient setting 3 times per week for 6 weeks. Assessments will take place before, after, and 1 month and 6 months after the completion of the training. A falls calendar will be kept by each participant for 6 months after completing the training to assess fall incidence (i.e., the number of falls, multiple falls and falls rate). In addition, we will measure gait under usual and dual task conditions, balance, community mobility, health related quality of life, user satisfaction and cognitive function.

Discussion: This randomized controlled trial will demonstrate the extent to which an intervention that combines treadmill training augmented by virtual reality reduces fall risk, improves mobility and enhances cognitive function in a diverse group of older adults. In addition, the comparison to an active control group that undergoes treadmill training without virtual reality will provide evidence as to the added value of addressing motor cognitive interactions as an integrated unit.
\end{abstract}

Trial Registration: (NIH)-NCT01732653

Keywords: Falls, Ageing, Gait, Cognitive function, Prevention, Virtual reality

\footnotetext{
* Correspondence: anatmi@tasmc.health.gov.l

'Department of Neurology, Laboratory for Gait Analysis \& Neurodynamics,

Movement Disorders Unit, Tel Aviv Sourasky Medical Center, 6 Weizmann

Street, Tel Aviv 64239, Israel

${ }^{2}$ School of Health Related Professions, Ben Gurion University, Beer Sheba,

Israel

Full list of author information is available at the end of the article
} 


\section{Background}

Gait impairments and falls are ubiquitous among older adults and patients with common neurological diseases. Approximately $30 \%$ of community-dwelling adults over the age of 65 fall at least once a year $[1,2]$. In persons with Parkinson's disease (PD), mild cognitive impairment (MCI) or dementia, falls are even more frequent with annual incidence rising to $60-80 \%[2,3]$. The consequences of these falls may be severe, leading to institutionalization, loss of functional independence, disability, fear of falling, depression and social isolation [4].

Most falls occur during walking [5,6] and, not surprisingly, gait impairment has been associated with an increased risk of falls [7,8]. Gait abnormalities in elderly fallers and patients with PD include reduced gait speed, stride length, and increased stride symmetry [9]. Fear of falling, cautious gait $[10,11]$, gait unsteadiness, or dysrhythmicity of stepping have also been recognized as mediators of fall risk [12-15].

There is a growing body of research that specifically links the cognitive sub-domains of attention and executive function (EF) to gait alterations and fall risk [15-21]. EF apparently plays a critical role in the regulation of gait especially under challenging conditions where decisions need to be made in real-time [22]. Walking while avoiding obstacles and walking while simultaneously performing another task, i.e., dual tasking (DT), place greater demands on cognitive resources such as divided attention and executive control, judgment, and reasoning, compared to "single task" walking [23-25]. EF scores and dual tasking gait performance have been associated with fall history and have been shown to predict future falls, even over several years of follow-up $[17,21,26]$. Although there is no universal agreement, many studies in patients with PD have reported that EF and dual tasking gait abilities are associated with fall risk [27-29] and attention-deficits predict future falls in patients with PD [30]. This may explain why falls occur so frequently among older adults, and even more so in patients with $\mathrm{PD}$ and patients with MCI. We suggest that these three groups share cognitive deficits that contribute to and exacerbate their fall risk. MCI patients are cognitively impaired, by definition. As much as $60 \%$ of patients who receive the diagnosis of $\mathrm{PD}$ already have cognitive deficits [31,32], and many older adults suffer from age-associated decline in cognitive function.

Another risk factor identified as a cause for falls in the elderly is obstacle crossing. Compared to healthy young adults, older adults walk more slowly during obstacle crossing [5,33-36], with smaller steps [34-36] landing dangerously closer to the obstacle with their lead limb [36-38]. Age-related deficits in vision, proprioception and visual-spatial orientation can also negatively impact postural stability and lower limb kinematics when crossing obstacles [5,34,36,37,39]. Obstacle negotiation heavily relies on the availability of ample cognitive resources, due to the need for motor planning and visually dependent gait regulation $[40,41]$.

Many intervention programs based on reported multiple risk factors have been proposed and evaluated to reduce fall risk [42]. However, despite the extensive knowledge on fall risk obtained in recent years, there is no consensus as to the most efficacious or optimal treatment approach $[43,44]$. Common treatments include exercise programs to improve strength or balance, educational programs, medication optimization, environmental modification and multi-factorial interventions involving a combination of several modalities. To date, however, the effects on fall risk tend to be small and the reported changes are largely focused on motor aspects with limited long-term retention [45-47].

Mahoney [48] suggested that perhaps the reason that multi-factorial interventions are not consistently successful is because they fail to address three major concepts: 1) training should be intensive, focused on the key impairment and become progressively more rigorous; 2) the training should fit the target population; 3) delivery of the intervention should include mechanisms to maximize motor learning and induce a behavioural change. We propose that insufficient focus on cognitive aspects, in particular, the motor-cognitive interactions that contribute to fall risk, might contribute to the suboptimal success of previous fall risk interventions. Even if cognitive function is targeted, it is generally done so in isolation and the motor-cognitive interactions are not directly addressed in an integrated fashion needed to successfully and safely ambulate in daily living.

To address this challenge, a multi-modal treadmill training program augmented by virtual reality (VR) (see Figure 1) was developed to deal with both the motor and cognitive aspects of fall risk and to promote motor learning critical for key tasks of safe ambulation. In general, VR is defined as a "high-end-computer interface that involves real time simulation and interactions through multiple sensorial channels" [49-51]. VR can be used to provide training in a more stimulating and enriching environment than traditional rehabilitation whilst providing feedback about performance to assist with learning new motor strategies of movement. Therefore, treadmill training augmented by VR is, theoretically, well-suited as a multi-factorial intervention for fall risk since it is designed to focus on the motor-cognitive aspects of fall risk such as dual tasking, obstacle negotiation and executive function.

In a pilot study [49], 20 patients with PD participated in an intervention based on a VR system for an obstacle navigation task. Patients walked on a treadmill while negotiating obstacles in a VR scene projected on a wall in 


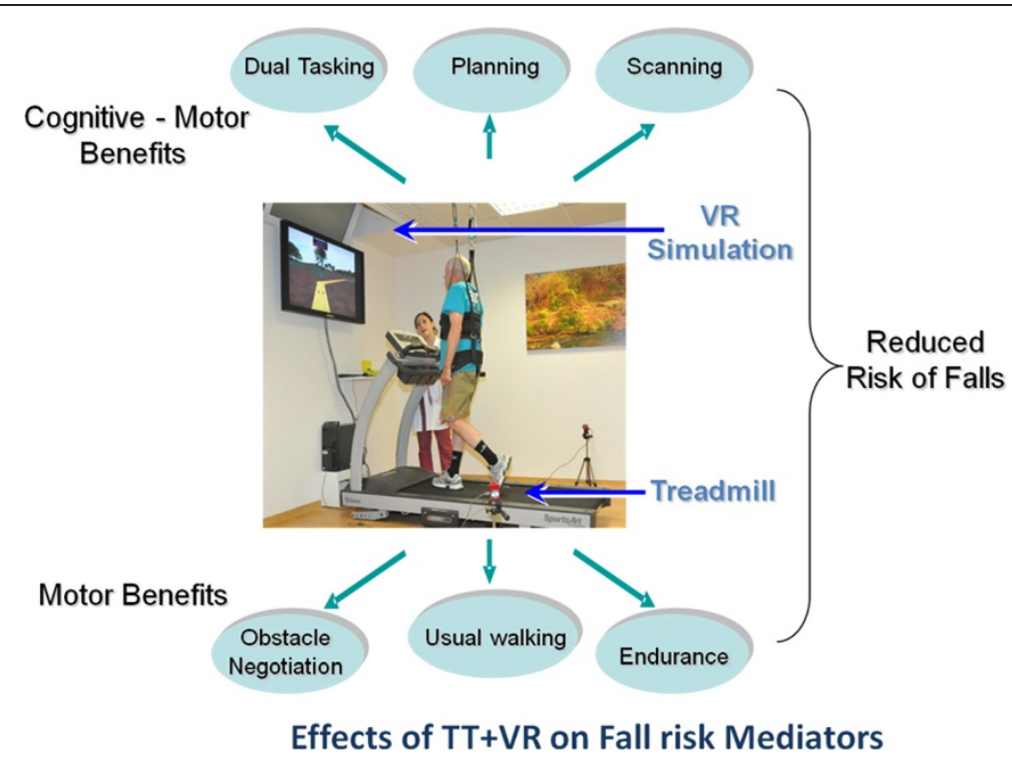

Figure 1 The V-TIME multi-modal intervention solution for reducing fall risk. Current treatment of fall risk focuses on motor, e.g., gait, problems. V-TIME focuses on both gait and cognitive deficits to optimally treat multiple, critical fall risk aspects and enhance mobility, physical activity and cognitive function. The current working version of V-TIME is shown. A patient trains on a treadmill while viewing a virtual environment that presents obstacles, different types of challenges, and feedback [49]. Written informed consent was obtained from the patient for publication of this case report and any accompanying images. A copy of the written consent is available for review by the Editor-in-Chief of this journal.

front of them. They trained for 3 times a week for 6 weeks for about 45 minutes in each session. Visual and auditory feedback was provided by the VR simulation upon error or success and at the end of each walk. After 6 weeks of training, comfortable gait speed significantly improved, as did stride length, gait variability, and overground obstacle negotiation. Dual task (DT) performance improved and there was evidence of enhanced task planning and set shifting. Increased gait speeds under all conditions (i.e., comfortable, fast, DT and six minute walk) were not only maintained at follow-up, but also continued to improve 4 weeks later, suggesting that the training generated a positive feedback loop that modified behaviour and overall mobility [49]. Encouraged by these results, an additional pilot study was carried out. Five elderly women who sustained at least 2 falls in the 6 months prior to the study trained using the same treadmill training with VR protocol. Here too, after training, improvements were observed in dual tasking, cognitive function, gait, and mobility, but perhaps the most promising finding was a decrease of $73 \%$ in the frequency of falls in the 6 months post-training as compared to 6 months pre-training [52].

The accumulating evidence on the importance of cognitive function to gait and falls combined with these initial findings formed the basis of the present study. The primary aim is to demonstrate that six weeks of treadmill training augmented by VR (TT $+\mathrm{VR})$ reduces the risk of falls in a relatively large and diverse group of older adults $(n=300)$, many of whom will likely have a spectrum of motor and cognitive deficits. The study will compare training effects of $\mathrm{TT}+\mathrm{VR}$ against an active control paradigm (TT without VR) in a randomized controlled trial. We hypothesize that a 6 week intervention with TT+VR compared to TT alone will reduce the incidence of falls and decrease the risk of falls in elderly adults, patients with PD and individuals with MCI. As a secondary question, we will also explore the neural correlates associated with dual task activation and any plastic effects resulting from the training using imaging techniques. However, protocols for these studies will not be presented in this manuscript.

\section{Methods/ Design \\ Design}

A prospective, single blinded, parallel group, randomized controlled trial (RCT) with 6 month follow-up will be employed. The study will include 300 older participants who have experienced two or more falls in the previous 6 months. Participants will be randomized to either the intervention or control group. The intervention group will receive 18 sessions of Treadmill Training with Virtual Reality (TT+VR) and the active control comparison will receive 18 training sessions of treadmill training alone (TT) without the VR simulation (see Figure 2). 


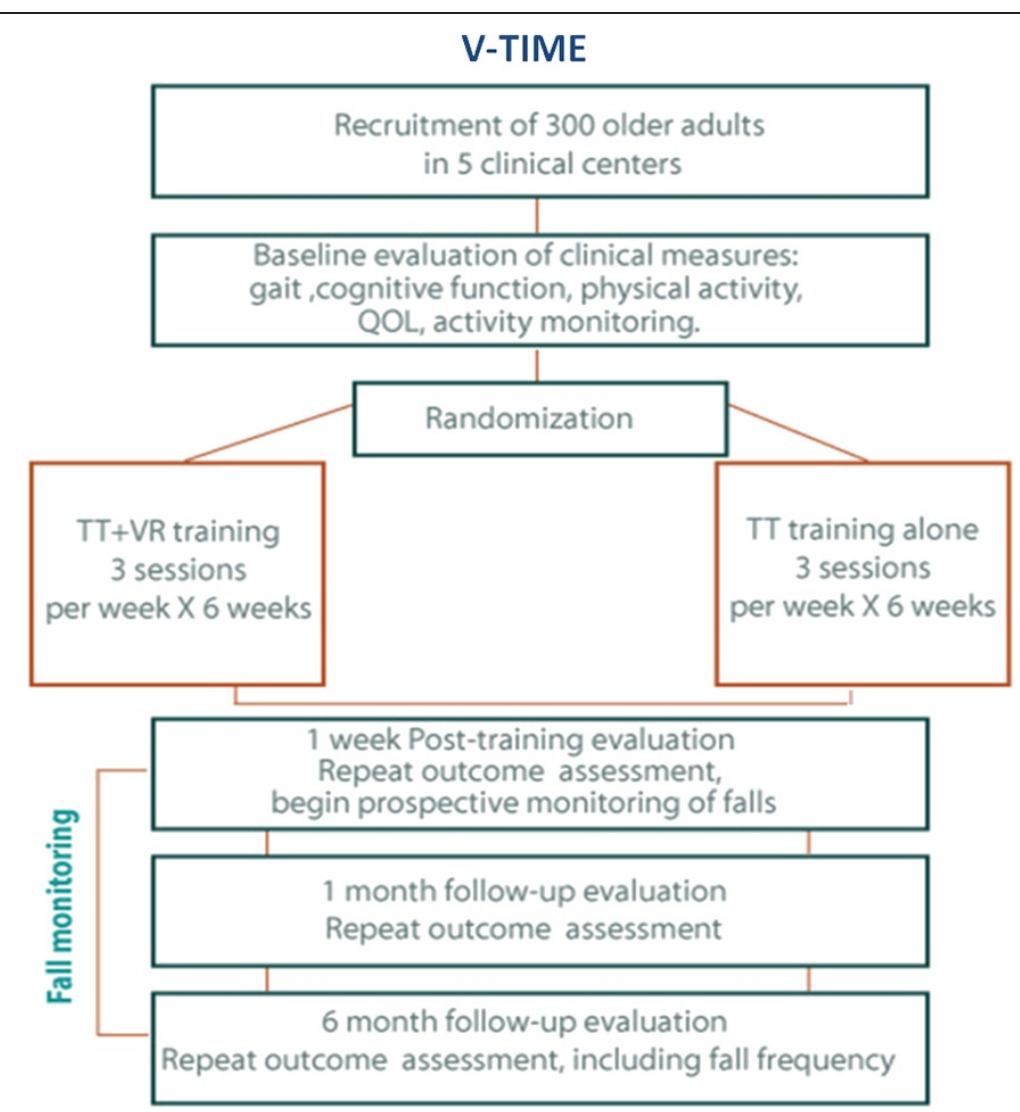

Figure 2 Summary of the study design and training protocol. $\pi$ : treadmill training. $T+V R$ : treadmill training augmented by the virtual reality simulation.

\section{Participants and setting}

The participants will be recruited from three groups: older adults with no cognitive impairment $(n=100)$; older adults with mild cognitive impairment (MCI) $(n=100)$ and people with Parkinson's disease (PD) with no cognitive impairment $(n=100)$. Subjects will be recruited if they meet the following criteria:

\section{Common inclusion criteria}

- 2 or more falls within 6 months prior to the beginning of the study

- Aged 60-85 years

- Able to walk for 5 minutes unassisted

- Adequate hearing (as evaluated by the whisper test) and vision capabilities (as measured using a Snellen chart).

- Stable medication for the past 1 month and anticipated over a period of 6 months

\section{Common exclusion criteria}

- Psychiatric co-morbidity (e.g., major depressive disorder as determined by DSM IV criteria)
- Clinical diagnosis of dementia or other severe cognitive impairment (Mini Mental State Exam score <24)

- History of stroke, traumatic brain injury or other neurological disorders (other than PD and MCI, for those groups)

- Acute lower back or lower extremity pain, peripheral neuropathy, rheumatic and orthopaedic diseases

- Unstable medical condition including cardiovascular instability in the past 6 months

- Unable to comply with the training or currently participating in another interfering therapy or a fall clinics program Interfering therapy

\section{Group specific criteria}

\section{Participants with PD Inclusion}

- Diagnosis of idiopathic PD, as defined by the UK Brain Bank criteria

- Hoehn and Yahr stage II-III 
- Taking anti-Parkinsonian medication

- Stable medication for past 1 month and anticipated over next 6 months or stable Deep Brain Stimulation for at least one month and expected following 6 months

\section{Exclusion}

- Severe freezing of gait (defined as having $>15$ on the new FOG questionnaire) [53]

\section{MCI group}

Inclusion

- Score 0.5 on the Clinical Dementia Rating Scale (CDR)

- Free from any neurological disorders that may have caused the cognitive impairment

\section{Sample size calculation}

The primary outcome measure is fall incidence rates during the 6 month post-training follow-up period. The sample size estimate is based on extrapolations from our pilot studies [49] and other related promising pilot work (e.g., Rosenblat et al. [54], Pai et al. [55] and, Weerdesteyn et al. [56]). Power was set at $80 \%$, alpha was set at $5 \%$ and we accounted for drop out rate of $20 \%$. Using a relatively conservative estimate, we assume that the control group fall incidence rate, after intervention, will be three falls per year. If we consider a $40 \%$ reduction for the treatment group relative to this, then, during the 6 month monitoring of falls incidence, a total of 166 subjects would be required for $80 \%$ power ( 83 in each group) to detect differences between the two treatment groups assuming non-inferiority with moderate correlations among covariates (R-squared $=0.50)$. A much smaller sample is needed to detect between group differences for the secondary outcomes. For example, with 22 subjects in the intervention and control arms, we will have $90 \%$ power to detect an intervention effect (assuming Cohen's $\mathrm{f}$ for ANOVA of 0.21), in dual tasking gait speed. To enhance our ability to examine the effects of the intervention on fall incidence within the three sub-groups (seniors, PD, MCI), we will aim to recruit 100 subjects per group, for a total of 300 subjects.

\section{Recruitment and randomization procedure}

The study will be conducted in 5 clinical centres across Europe (Lab for Gait \& Neurodynamics, Tel Aviv Sourasky Medical Centre, Israel; Department of Rehabilitation Sciences, Katholieke Universiteit Leuven, Belgium; Institute for Ageing and Health, Newcastle University UK; Department of Neurosciences, University of Genoa, Italy; Departments of Geriatric Medicine \&
Neurology, Radboud University Nijmegen Medical Centre, The Netherlands).

Ethical approval was obtained by ethics committees of each of the above clinical sites. Eligible subjects, who agree to participate, will be asked to provide an informed written consent after which they will be randomized to one of two arms of the study: 1) TT+VR; 2) TT. A permuted blocked randomization procedure will be used selecting randomly from a block size of 4, 6 or 8 . Group allocation will be performed by a third party not involved in the day to day running of the study; the treating therapist will be notified by e-mail to ensure concealed allocation.

\section{Intervention}

All interventions will be delivered by therapists trained in the standard protocols across centres in the consortium countries. Consistent with the motor learning literature and the pilot studies $[49,52]$, all subjects will be trained 3 times a week for 6 weeks, each session will last approximately 45 minutes.

\section{Virtual reality system}

Details of the instrumentation are provided elsewhere $[49,52]$. Briefly, the system includes a camera based motion capture (Kinect) and a computer generated simulation. The camera will be used to collect the movement of the participant's feet while walking on the treadmill. These images will be transferred into the computer simulation and projected to the patient on a large screen while training, enabling the subjects to see their feet walking within the VR simulation. The virtual environment (VE) will consist of obstacles, different pathways, narrow corridors and distracters, requiring modulations of step amplitude in two planes (i.e., height and width) coordinated with walking behaviour. The speed, orientation, size, frequency of appearance and shape of the targets will be manipulated according to individual needs following a standardized protocol. Environmental features (e.g., visibility, settings and distractions) will be adjusted to increase training complexity. The VE will impose a cognitive load requiring attention and response selection as well as processing of rich visual stimuli involving several perceptual processes. The system will provide visual and auditory feedback of successful or unsuccessful task performance to enhance motor learning. Adaptability of the system is foreseen to adjust training parameters to the clinical needs of the individual participant.

\section{$T T+V R$ group (the intervention group) Motor aspect of training}

Gait speed over-ground will be measured over 10 meters at the beginning of each week of training. This speed 
will be registered and the treadmill speed will be set accordingly, as detailed below. Training will be divided into bouts of walking and rest breaks in between. The duration of the initial session should be ideally 20 minutes of walking time. A safety harness will be attached to an over head suspension system, but no weight support will be provided.

\section{Training progression}

Training progression will be based on increasing both motor and cognitive challenges, individualized to the participant's level of performance. The motor component of training progression will include an increase in the treadmill speed and duration of training. Treadmill speed in the first week will be set at $80 \%$ of over ground gait speed. In the second week, the treadmill speed will increase to $90 \%$ of over ground speed. Another 10minutes in duration will be added. From the third week, the speed goal will increase by $10 \%$ every week and 1 minute of walking will be added to each of the walking bouts (a total of an additional 3-5 minutes per session compared to the previous session). This progression is subject to the performance and the patient's ability.

The cognitive components of training progression will include changing the number, size and shape of obstacles, and the frequency, speed and direction at which they appear. The Virtual Environment characteristics will also be manipulated by reducing visibility and adding distracters (e.g., birds, cars). During week 1, obstacles will appear infrequently (e.g., every 30 seconds, at low level of difficulty), be unilateral in direction and the environmental features will be minimal (i.e., high visibility, day time walking, minimal distracters). Based on the subject's performance in weeks 2 and 3, the frequency of appearance of the obstacles will increase, obstacles will appear on the more challenged side and their features (horizontal vs. vertical) will be individualized. Environmental features will appear with some minimal distracters during weeks 2 and 3 . In week 4 , subjects will be introduced to a new environment to allow for more diversity in training and to maximize transfer to the real-world. Throughout, training should maintain the ratio of 80:20 success/failure rates in order to enhance motor learning. If subjects are not successful, the difficulty level will be decreased to the level previously achieved and vice versa.

\section{Treadmill training (TT) (active control) group}

The participants will walk on the treadmill without receiving the feedback from the VR. As in the TT+VR group, their gait speed over-ground will be measured at the beginning of each week of training. Progression and the time spent with the trainer will follow the same guidelines as the motor progression of the TT+VR group and will include increasing the duration of each of the walking bouts and increasing walking speed.

\section{Assessment protocol}

A repeated measures design will be employed with assessments performed 1 week pre-training, posttraining, and at 1 month and 6 months post intervention (Table 1). A trained assessor in each centre, not involved in training and blinded to group allocation, will perform all assessments. Each participant will be assessed at about the same time of day to avoid variability of performance due to any circadian rhythms or medication intake cycle.

\section{Outcome measures}

\section{Primary outcome measures}

The primary outcome measure of the study is fall rate. Participants will keep a falls calendar for 6 months post intervention. Consistent with the recommendations of the Prevention of Falls Network Europe (ProFaNE), a fall will be defined as "an unexpected event in which the participant comes to rest on the ground, floor or lower level". Each time the participant falls he/she will tick the date on the calendar. These calendars will be returned to the researchers every two weeks in a pre-addressed envelope or using an online electronic calendar. Periodic contact by the research staff with each participant will be used to maximize compliance with the fall calendars.

\section{Secondary outcome measures}

Gait Gait speed and gait variability under usual and DT conditions and while negotiating physical obstacles will be measured. Participants will be asked to walk in a well-lit corridor under 3 conditions each of 1 minute: i) walking in a comfortable speed, ii) walking while subtracting $3 \mathrm{~s}$ from a predefined number (dual task), iii) walking while negotiating two obstacles placed on the floor at specific locations. The GaitRite mat, a sensorized 7 meter carpet (CIR Systems, Inc. Haverton MA), will capture individual footfall data using embedded pressure sensors. This is a valid and reliable method of assessing the spatiotemporal parameters of gait in healthy older adults and in patients with Parkinson's disease [57]. Spatiotemporal gait characteristics (e.g., gait speed $(\mathrm{m} / \mathrm{s})$, stride length (m), stride time (s), swing time (\%), asymmetry, and step width $(\mathrm{cm})$ ) will be determined. Overground obstacle negotiation will be evaluated by placing physical obstacles on the GaitRite. The distance between the heel and the physical obstacle during the loading response of the lead foot will be measured to assess clearance and efficient obstacle negotiation.

Small, lightweight 3 axial accelerometers (APDM, Oregon, USA) will be worn on both feet, both wrists 
Table 1 Assessment of outcome measures across the protocol

\begin{tabular}{|c|c|c|c|c|c|c|}
\hline & Category & Outcome measures & $\begin{array}{c}\text { Pre } \\
\text { training }\end{array}$ & $\begin{array}{l}\text { Post } \\
\text { training }\end{array}$ & $\begin{array}{l}\text { One month } \\
\text { follow up }\end{array}$ & $\begin{array}{l}\text { Six month } \\
\text { follow up }\end{array}$ \\
\hline $\begin{array}{l}\text { Primary outcome } \\
\text { measure }\end{array}$ & Falls & Fall frequency & & & & $x$ \\
\hline \multirow{14}{*}{$\begin{array}{l}\text { Secondary outcome } \\
\text { measures }\end{array}$} & \multirow[t]{3}{*}{ Gait } & Gait speed & $x$ & $x$ & $x$ & $x$ \\
\hline & & Gait variability & $x$ & $x$ & $x$ & $x$ \\
\hline & & $2 \mathrm{MWT}$ & $x$ & $x$ & $x$ & $x$ \\
\hline & \multirow[t]{4}{*}{ Balance and mobility } & FSST & $x$ & $x$ & $x$ & $x$ \\
\hline & & SPPB & $x$ & $x$ & $x$ & $x$ \\
\hline & & mini-BEST & $x$ & $x$ & $x$ & $x$ \\
\hline & & Community ambulation & $x$ & $x$ & $x$ & \\
\hline & \multirow[t]{4}{*}{ Cognitive function } & MoCA & $x$ & & & \\
\hline & & TMT & $x$ & $x$ & $x$ & $x$ \\
\hline & & $\begin{array}{l}\text { Mindstreams tests of cogntive } \\
\text { function }\end{array}$ & $x$ & $x$ & & $x$ \\
\hline & & Verbal Fluency & $x$ & $x$ & $x$ & $x$ \\
\hline & \multirow{2}{*}{$\begin{array}{l}\text { Healthy Related Quality } \\
\text { of Life }\end{array}$} & SF-36 & $x$ & $x$ & $x$ & $x$ \\
\hline & & FES-I & $x$ & $x$ & $x$ & $x$ \\
\hline & $\begin{array}{l}\text { User satisfaction and } \\
\text { views }\end{array}$ & User satisfaction Questionnaire & & $x$ & & \\
\hline
\end{tabular}

2MWT- 2 Minute Walk Test, FSST-Four Square Step Test, SPPB- Short Physical Performance Battery, mini-BEST- The mini-Balance Evaluation Systems Test, MOCAMontreal Cognitive Assessment, TMT- Trail Making Test, FES-I- Fall Efficacy Scale International.

and on the lower back of the participants during all gait measurements to quantify temporal measures such as stride time and gait variability [8]. Gait variability (i.e., the inconsistency from one stride to the next) will be determined by calculating the magnitude of stride-to -stride fluctuations, normalized to each subject's mean stride time to define the Coefficient of Variation (Coefficient of Variation $(\mathrm{CV}=$ (standard deviation/mean) $\times$ 100)). Gait variability is a validated and reliable measure reflecting fall risk that has been used with patients with PD [58], older adults [24] and individuals with MCI [59]. Data will be collected at $240 \mathrm{HZ}$, saved onto a computer and analysed using proprietary software.

Endurance will be assessed using the 2 Minute Walk Test. This performance-based tool was originally developed to assess exercise tolerance among individuals with respiratory disease, but has shown high test retest reliability and validity in assessment of gait endurance in older adults [60] and in individuals with neurological conditions $[61,62]$.

\section{Balance and mobility}

The Four Square Step Test (FSST) requires subjects to rapidly change direction while stepping forward, backward, and sideway, over a low obstacle. Time to complete the test is measured. The test has been validated in older adults [63] with sensitivity of $85 \%$ and specificity of $88-100 \%$ in predicting fall risk [63].
The Short Physical Performance Battery (SPPB) consists of three types of physical maneuvers: the balance tests, the gait speed test, and the chair stand test. The SPPB is highly reliable in older adults $(\mathrm{ICC}=0.83$ 0.89 ) and has demonstrated a strong and consistent association with health status measures, in spite of the socioeconomic and cultural differences [64].

The mini-Balance Evaluation Systems Test (miniBESTest) is a performance based measure differentiating balance problems into 6 underlying systems that may be impaired: biomechanical, stability limits, postural responses, anticipatory postural adjustments, sensory orientation, dynamic balance during gait and cognitive effects. The mini-BESTest has been shown to be a reliable (ICC $=0.91$ ) and valid measure of balance in individuals with PD [65].

Community ambulation will be assessed using 1) the Physical Activity Scale for the Elderly (PASE). This 27 item self report questionnaire assess habitual physical activity in the home and community environment. The questionnaire was designed to address cultural differences, is available in multiple languages and has been validated for older adults [66]; 2) a tri-axial accelerometer (Axivity Ltd.) will be worn by the participants for 7 days to quantify and monitor stepping and physical activity. The device which records at $100 \mathrm{~Hz}$ will be mounted on the trunk (L5) and will derive the following outcome measures: step count, postural transitions, sedentary time, percentage walking time, number and time 
of walking and sedentary bouts. Data will be obtained one-week pre and post training.

\section{Cognitive function}

Cognitive function will be assessed using a computerized neuropsychological test battery (Mindstreams ${ }^{\circledR}$, NeuroTrax Corp., NJ) [67]. The battery assesses different cognitive domains including memory, attemtion, executive function, visual spatial processing and a global cognitive composite. The test battery generates age and education adjusted composite indices of each cognitive domain on an IQ like scale, with the score of 100 representing the estimated population mean normalized for age and education level. The battery has been validated in elderly adults, patients with mild cognitive impairment, and patients with PD and has shown to be useful in predicting falls $[17,21,68-70]$.

In addition, we will also include standardized neuropsychological tests such as the Montreal Cognitive Assessment (MoCA); a rapid screening instrument for global cognitive dysfunction. Different cognitive domains are assessed (attention and concentration, executive functions, memory, language, visuo-constructional skills, conceptual thinking, calculations, and orientation). The MOCA was found to be a valid instrument for cognitive screening in $\mathrm{MCI}$ and PD [71,72]. In this study the MoCA will be used as a descriptive measure.

The Trail Making Test (TMT) is a neuropsychological test of visual attention and task switching. It consists of two parts in which the subject is instructed to connect a set of 25 dots as fast as possible while still maintaining accuracy. The test provides information about visual search speed, scanning, speed of processing, mental flexibility, and executive functioning. The TMT is valid and reliable for older adults $[73,74]$ and has been previously associated with decreased gait speed, dual task activity, and obstacle clearance [75].This is a well suited outcome measure given the nature of the training.

Verbal Fluency is a test of working memory and language in which participants have to say as many words as possible from a category in a given time (usually 60 seconds). The test includes both semantic and phonemic sections, is related to executive function, and has been shown to be highly reliable and valid in the elderly population [76].

\section{Health-related quality of life}

The SF-36 Health Survey is a generic self-report questionnaire designed to address health related quality of life. The SF-36 includes one multi-item scale measuring several constructs such as physical functioning; bodily pain; social functioning; general mental health (psychological distress and psychological wellbeing); vitality (energy/fatigue); and general health perceptions. Criterion validity has been established but the scores could also be divided into two aggregate summary measures; the Physical Component Summary (PCS) and the Mental Component Summary (MCS). SF-36 has been validated for older adults and patients with PD $[77,78]$.

Fear of Falling will be evaluated using the Falls Efficacy Scale-International. The FES-I has been significantly associated with performance-based measures of balance and mobility including gait speed and medial-lateral sway. The scale has been shown to be sensitive to change in older adults with and without cognitive impairments $[79,80]$.

\section{User satisfaction and views}

A questionnaire was developed by the researchers to assess the satisfaction of the participants from the training and to try and obtain subjective information regarding the usability and efficacy of such an intervention in reducing fear of falling, fall risk and frequency of falls.

\section{Data analysis}

Statistical analysis will be undertaken using SPSS version 19.0 (SPSS Corp, Chicago, IL, USA). All analysis will be conducted on an intention-to-treat principle using all randomized participants. Demographic characteristics and baseline data will be summarized by descriptive statistics using means, standard deviations and 95\% confidence intervals for continuous variables, median and inter-quartile ranges for non-normal continuous or ordinal data and percentages for categorical data, and will be evaluated for normalcy and homogeneity. For the primary outcome measure, fall rate will be analyzed by calculating relative risk using negative bionomial regression models that adjust for any potential confounders [81]. Fall rate and fall status (none faller, faller and multiple faller) will then be compared within and between groups. The secondary outcome measures will be analyzed using repeated measures analysis of variance (RMANOVA) to assess differences between groups (intervention) and across time (follow up) for each group of participants and then compared across groups. All data will be adjusted for multiple comparisons.

\section{Safety considerations and adverse events}

All measurements are non-invasive and place the subject at no risk other than those that normally may occur during walking. For some of the patients, in particular those who were not practicing any kind of physical exercise prior to the intervention; there is a slight possibility that subjects might feel some muscle soreness and fatigue after training. To prevent excessive fatigue, subjects will be encouraged to take breaks as needed throughout all study procedures. In addition, the study was designed for gradual increases in intensity which will help to increase endurance and build muscle strength. Virtual 
reality may cause cyber sickness, a sensation similar to motion sickness. This phenomenon is, however, very rare and is related to highly immersive technology. The proposed study will use a $2 \mathrm{D}$ projection to deliver the VR simulation which decreases the risk of developing cyber sickness.

\section{Discussion}

The aim of this study is to establish a practical and feasible solution for enhancing mobility, preventing falls and reducing disability among diverse groups of older adults using a unique intervention that combines treadmill training and virtual reality. What sets this project apart from previous work in this field is that the study simultaneously addresses both motor and cognitive function and their interactions that are key to falls using a large, RCT study design, with an active comparison control for assessing efficacy. Training is provided in a virtual environment that implicitly challenges, teaches, and enhances visual scanning, planning, DT abilities and obstacle negotiation. The additional training goals that aim to enhance the cognitive aspects of mobility have not yet been integrated into common practice and are one of the important added features of the proposed intervention. The unique training program takes onboard all aspects of motor learning in that it probes retention as well as transfer of training to real-world activities to maximize resilient effects.

In a sense, this study also addresses the concepts and concerns raised by Mahoney et al. [48]. The proposed training is intensive, focuses on the key impairment and becomes progressively more rigorous. The training and protocol were designed to meet the needs of a diverse group of older adults including those with cognitive deficits and motor impairment due to neurodegeneration. The intervention maximizes motor learning in order to induce a behavioural change. Moreover, training in the computer-controlled virtual environment makes the therapy and protocol standardized and reproducible.

The training protocol that is at the basis of this study was developed based on recently established guidelines on complex interventions in geriatrics [82-87]. The proposed protocol is based on the needs of the three groups who share a high risk of falls, in part due to cognitive deficits. Focus groups and questionnaires have been used to refine the intervention. Feasibility of using such an intervention was assessed and pilot studies were carried out. Further, the outcome measures are validated and selected to evaluate the effects of the intervention on falls and the motor cognitive interactions that contribute to fall risk. This process enables us to confidently advance into a large randomized controlled trial to explore efficacy in comparison to an active training control group.

Evidence on the efficacy of fall prevention in geriatrics is not yet ideal and large randomized control trials are needed. To promote motor learning required for safe ambulation, fall prevention interventions should include motor and cognitive aspects relating to falls, taskspecific and generalized training, with the intervention centred around the user's needs. The proposed intervention set out to bridge all these needs. The knowledge that will be generated by the results of this study are likely to inform new models of care that combine technology, mobility training, and cognitive remediation to reduce risk of falls and enhance mobility even in a chronic disease profile.

\section{Conclusions}

This randomized controlled trial will demonstrate the extent to which an intervention that combines treadmill training augmented by virtual reality reduces fall risk, improves mobility and enhances cognitive function in a diverse group of older adults. In addition, the comparison to an active control group that undergoes treadmill training without the added virtual reality will provide evidence as to the added value of an intervention that addresses motor cognitive interactions as an integrated unit.

\section{Competing interests}

All authors declare that they have no competing interests.

\section{Authors' contributions}

AM and JMH participated in designing the study, designing the VR system to be used, writing and reviewing of the manuscript. LR participated in designing the study and writing and reviewing the manuscript. MR, FN and $\mathrm{KD}$ participated in reviewing the manuscript, EP, GA and AN participated in designing the study and reviewing the manuscript. All authors read and approved the final manuscript.

\section{Acknowledgments}

Besides the clinical partners, V-TIME also includes technical partners without which the project would not be successful. We would like to thank our partners from the University of Sassari in Sardinia (UNISS), the partners from Inition 3D technologies (INITION), Advanced Drug Development Services (ADDS) and Beacon Tech Limited (BTL) for their contribution. The project was funded by the European Commission (FP7 project V-TIME- 278169).

\section{Author details}

${ }^{1}$ Department of Neurology, Laboratory for Gait Analysis \& Neurodynamics, Movement Disorders Unit, Tel Aviv Sourasky Medical Center, 6 Weizmann Street, Tel Aviv 64239, Israel. ${ }^{2}$ School of Health Related Professions, Ben Gurion University, Beer Sheba, Israel. ${ }^{3}$ Institute for Aging and Health, University of Newcastle, Newcastle, UK. ${ }^{4}$ Department of Geriatric Medicine and Neurology, Radboud University Nijmegen Medical Center, Nijmegen, The Netherlands. ${ }^{5}$ Department of Neurosciences, Universita Degli Studi Di Genova, Genova, Italy. ${ }^{6}$ Department of Rehabilitation Science, Katholieke Universiteit Leuven, Leuven, Belgium. ${ }^{7}$ Department of Physical Therapy, Sackler Faculty of Medicine, Tel-Aviv University, Tel-Aviv, Israel. ${ }^{8}$ Harvard Medical School, Boston, MA, USA.

Received: 26 August 2012 Accepted: 15 January 2013 Published: 6 February 2013 


\section{References}

1. Blake AJ, Morgan K, Bendall MJ, et al: Falls by elderly people at home: prevalence and associated factors. Age Ageing 1988, 17(6):365-372

2. Tinetti ME, Speechley M, Ginter SF: Risk factors for falls among elderly persons living in the community. N Engl J Med 1988, 319(26):1701-1707.

3. Wood BH, Bilclough JA, Bowron A, Walker RW: Incidence and prediction of falls in Parkinson's disease: a prospective multidisciplinary study. J Neurol Neurosurg Psychiatry 2002, 72(6):721-725.

4. Rubenstein LZ, Josephson KR: Falls and their prevention in elderly people: what does the evidence show? Med Clin North Am 2006, 90(5):807-824.

5. Mackenzie L, Byles J, D'Este C: Validation of self-reported fall events in intervention studies. Clin Rehabil 2006, 20(4):331-339.

6. Sartini M, Cristina ML, Spagnolo AM, et al: The epidemiology of domestic injurious falls in a community dwelling elderly population: an outgrowing economic burden. Eur J Public Health 2010, 20(5):604-606.

7. Axer H, Axer M, Sauer H, Witte OW, Hagemann G: Falls and gait disorders in geriatric neurology. Clin Neurol Neurosurg 2010, 112(4):265-274.

8. Hausdorff JM, Rios D, Edelberg HK: Gait variability and fall risk in community-living older adults: a 1-year prospective study. Arch Phys Med Rehabil 2001, 82:1050-1056

9. Auvinet B, Berrut $G$, Touzard C, et al: Reference data for normal subjects obtained with an accelerometric device. Gait Posture 2002, 16(2):124-134.

10. Ashburn A, Stack E, Pickering RM, Ward CD: A community-dwelling sample of people with Parkinson's disease: characteristics of fallers and nonfallers. Age Ageing 2001, 30(1):47-52.

11. Legters K: Fear of falling. Phys Ther 2002, 82(3):264-272.

12. Hausdorff JM, Nelson ME, Kaliton D, et al: Etiology and modification of gait instability in older adults: a randomized controlled trial of exercise. J Appl Physiol 2001, 90(6):2117-2129.

13. Hausdorff JM, Yogev G, Springer S, Simon ES, Giladi N: Walking is more like catching than tapping: gait in the elderly as a complex cognitive task. Exp Brain Res 2005, 164(4):541-548.

14. Hausdorff JM, Yogev G: Cognitive function may be important for fall injury prevention trials. J Am Geriatr Soc 2006, 54(5):865-866.

15. Hausdorff JM, Doniger GM, Springer S, Yogev G, Giladi N, Simon ES: A common cognitive profile in elderly fallers and in patients with Parkinson's disease: the prominence of impaired executive function and attention. Exp Aging Res 2006, 32(4):411-429.

16. Allcock LM, Rowan EN, Wesnes K, Kenny RA, Burn DJ: Impaired attention: a risk factor for falls in Parkinson's disease? Mov Disord 2007, 22:\$204.

17. Herman T, Mirelman A, Giladi N, Schweiger A, Hausdorff JM: Executive control deficits as a prodrome to falls in healthy older adults: a prospective study linking thinking, walking, and falling. J Gerontol A Biol Sci Med Sci 2010, 65(10):1086-1092.

18. Holtzer R, Friedman R, Lipton RB, Katz M, Xue X, Verghese J: The relationship between specific cognitive functions and falls in aging Neuropsychology 2007, 21(5):540-548.

19. Holtzer R: Central control of mobility: evidence from cognitive, genetic and functional neuroimaging studies. Parkinsonism Relat Disord 2010 16:S4-S5.

20. Liu-Ambrose T, Katarynych LA, Ashe MC, Nagamatsu LS, Hsu CL: Dual-task gait performance among community-dwelling senior women: the role of balance confidence and executive functions. J Gerontol A Biol Sci Med Sci 2009, 64(9):975-982

21. Springer S, Giladi N, Peretz C, Yogev G, Simon ES, Hausdorff JM: Dualtasking effects on gait variability: the role of aging, falls, and executive function. Mov Disord 2006, 21(7):950-957.

22. Ble A, Volpato S, Zuliani G, et al: Executive function correlates with walking speed in older persons: The InCHIANTI study. J Am Geriatr SoC 2005, 53(3):410-415.

23. Sheridan PL, Hausdorff JM: The role of higher-level cognitive function in gait: executive dysfunction contributes to fall risk in Alzheimer's disease. Dement Geriatr Cogn Disord 2007, 24(2):125-137.

24. van lersel MB, Kessels RP, Bloem BR, Verbeek AL, Olde Rikkert MG: Executive functions are associated with gait and balance in community-living elderly people. J Gerontol A Biol Sci Med Sci 2008, 63(12):1344-1349.

25. Yogev-Seligmann G, Hausdorff JM, Giladi N: The role of executive function and attention in gait. Mov Disord 2008, 23(3):329-342.

26. Muir SW, Gopaul K, Montero Odasso MM: The role of cognitive impairment in fall risk among older adults: a systematic review and meta-analysis. Age Ageing 2012, 41(3):299-308.
27. Camicioli R, Howieson D, Lehman S, Kaye J: Talking while walking: the effect of a dual task in aging and Alzheimer's disease. Neurology 1997 48(4):955-958

28. Hausdorff JM, Balash J, Giladi N: Effects of cognitive challenge on gait variability in patients with Parkinson's disease. J Geriatr Psychiatry Neurol 2003, 16(1):53-58

29. Yogev G, Giladi N, Peretz C, Springer S, Simon ES, Hausdorff JM: Dual tasking, gait rhythmicity, and Parkinson's disease: which aspects of gait are attention demanding? Eur J Neurosci 2005, 22(5):1248-1256.

30. Allcock LM, Rowan EN, Steen IN, Wesnes K, Kenny RA, Burn DJ: Impaired attention predicts falling in Parkinson's disease. Parkinsonism Relat Disord 2009, 15(2):110-115.

31. Aarsland D, Bronnick K, Larsen JP, Tysnes OB, Alves G: Cognitive impairment in incident, untreated Parkinson disease: the Norwegian ParkWest study. Neurology 2009, 72(13):1121-1126.

32. Pagonabarraga J, Kulisevsky J: Cognitive impairment and dementia in Parkinson's disease. Neurobiol Dis 2012, 46(3):590-596.

33. Chapman GJ, Hollands MA: Evidence that older adult fallers prioritise the planning of future stepping actions over the accurate execution of ongoing steps during complex locomotor tasks. Gait Posture 2007 26(1):59-67.

34. Chen HC, Schultz AB, Ashton-Miller JA, Giordani B, Alexander NB, Guire KE: Stepping over obstacles: dividing attention impairs performance of old more than young adults. J Gerontol A Biol Sci Med Sci 1996 51(3):M116-M122.

35. Di Fabio RP, Greany JF, Zampieri C: Saccade-stepping interactions revise the motor plan for obstacle avoidance. J Mot Behav 2003, 35(4):383-397.

36. Lowrey CR, Watson A, Vallis LA: Age-related changes in avoidance strategies when negotiating single and multiple obstacles. Exp Brain Res 2007, 182(3):289-299.

37. Galna B, Murphy AT, Morris ME: Obstacle crossing in people with Parkinson's disease: foot clearance and spatiotemporal deficits. Hum Mov Sci 2010, 29(5):843-852

38. Galna B, Lord S, Daud D, Archibald N, Burn D, Rochester L: Visual sampling during walking in people with Parkinson's disease and the influence of environment and dual-task. Brain Res 2012, 1473:35-43.

39. Menant JC, St George RJ, Fitzpatrick RC, Lord SR: Impaired depth perception and restricted pitch head movement increase obstacle contacts when dual-tasking in older people. J Gerontol A Biol Sci Med Sci 2010, 65(7):751-757.

40. Brown LA, McKenzie NC, Doan JB: Age-dependent differences in the attentional demands of obstacle negotiation. J Gerontol A Biol Sci Med Sci 2005, 60(7):924-927.

41. Sparrow WA, Bradshaw EJ, Lamoureux E, Tirosh O: Ageing effects on the attention demands of walking. Hum Mov Sci 2002, 21(5-6):961-972.

42. AGS Guidelines: Guideline for the prevention of falls in older persons. American Geriatrics Society, British Geriatrics Society, and American Academy of Orthopaedic Surgeons Panel on Falls Prevention. J Am Geriatr Soc 2001, 49(5):664-672.

43. Gillespie LD, Robertson MC, Gillespie WJ, et al: Interventions for preventing falls in older people living in the community. Cochrane Database Syst Rev 2009, 2:CD007146.

44. Hauer K, Becker C, Lindemann U, Beyer N: Effectiveness of physical training on motor performance and fall prevention in cognitively impaired older persons: a systematic review. Am J Phys Med Rehabil 2006, 85(10):847-857.

45. Goodwin VA, Richards SH, Taylor RS, Taylor AH, Campbell JL: The effectiveness of exercise interventions for people with parkinson's disease: a systematic review and meta-analysis. Mov Disord 2008, 23(5):631-640.

46. Gillespie LD, Gillespie WJ, Robertson MC, Lamb SE, Cumming RG, Rowe BH: Interventions for preventing falls in elderly people. Cochrane Database Syst Rev 2003, 4:CD000340.

47. Kannus P, Parkkari J, Koskinen $S$, et al: Fall-induced injuries and deaths among older adults. JAMA 1999, 281(20):1895-1899.

48. Mahoney JE: Why multifactorial fall-prevention interventions may not work: Comment on "Multifactorial intervention to reduce falls in older people at high risk of recurrent falls". Arch Intern Med 2010, 170(13):1117-1119.

49. Mirelman A, Maidan I, Herman T, Deutsch JE, Giladi N, Hausdorff JM: Virtual reality for gait training: can it induce motor learning to enhance 
complex walking and reduce fall risk in patients with Parkinson's disease? J Gerontol A Biol Sci Med Sci 2010,

50. Burdea GC: Virtual rehabilitation-benefits and challenges. Methods Inf Med 2003, 42(5):519-523.

51. de Bruin ED, Schoene D, Pichierri G, Smith ST: Use of virtual reality technique for the training of motor control in the elderly. Some theoretical considerations. Z Gerontol Geriatr 2010, 43(4):229-234.

52. Mirelman A, Raphaeli Baer N, Dorfman M, Brozgol M, Giladi N, Hausdorff JM: Virtual Reality in Idiopathic fallers - A pilot study. Zurich: Conference proceedings from the International Conference for Virtual Reality; 2011

53. Nieuwboer $A$, Rochester $L$, Herman $T$, et al: Reliability of the new freezing of gait questionnaire: agreement between patients with Parkinson's disease and their carers. Gait Posture 2009, 30(4):459-463.

54. Grabiner MD, Bareither ML, Gatts S, Marone J, Troy KL: Task-specific training reduces trip-related fall risk in women. Med Sci Sports Exerc 2012, 44(12):2410-4.

55. Pai YC, Bhatt T, Wang E, Espy D, Pavol MJ: Inoculation against falls: rapid adaptation by young and older adults to slips during daily activities. Arch Phys Med Rehabil 2010, 91(3):452-459.

56. Weerdesteyn V, Rijken H, Geurts AC, Smits-Engelsman BC, Mulder T, Duysens $\mathrm{J}$ : A five-week exercise program can reduce falls and improve obstacle avoidance in the elderly. Gerontology 2006, 52(3):131-141.

57. Bilney $B$, Morris $M$, Webster $K$ : Concurrent related validity of the GAITRite walkway system for quantification of the spatial and temporal parameters of gait. Gait Posture 2003, 17(1):68-74

58. Hausdorff JM, Cudkowicz ME, Firtion R, Wei JY, Goldberger AL: Gait variability and basal ganglia disorders: stride-to-stride variations of gait cycle timing in Parkinson's disease and Huntington's disease. Mov Disord 1998, 13(3):428-437.

59. Montero-Odasso M, Bergman $\mathrm{H}$, Phillips NA, Wong $\mathrm{CH}$, Sourial N, Chertkow $\mathrm{H}$ : Dual-tasking and gait in people with mild cognitive impairment. The effect of working memory. BMC Geriatr 2009, 9:41.

60. Brooks D, Davis AM, Naglie G: Validity of 3 physical performance measures in inpatient geriatric rehabilitation. Arch Phys Med Rehabil 2006, 87(1):105-110

61. Light KE, Behrman AL, Thigben M, Triggs WJ: The 2-minute walk test: a tool for evaluating endurance in clients with Parkinson's disease. Neurol Rep 1997, 21:136-139.

62. Rossier P, Wade DT: Validity and reliability comparison of 4 mobility measures in patients presenting with neurologic impairment. Arch Phys Med Rehabil 2001, 82(1):9-13.

63. Dite $W$, Temple VA: A clinical test of stepping and change of direction to identify multiple falling older adults. Arch Phys Med Rehabil 2002, 83(11):1566-1571.

64. Freire AN, Guerra RO, Alvarado B, Guralnik JM, Zunzunegui MV: Validity and reliability of the short physical performance battery in two diverse older adult populations in quebec and Brazil. J Aging Health 2012, 24(5):863-878

65. King LA, Priest KC, Salarian A, Pierce D, Horak FB: Comparing the MiniBESTest with the berg balance scale to evaluate balance disorders in Parkinson's disease. Parkinsons Dis 2012, 2012:375419.

66. Craig CL, Marshall AL, Sjostrom M, et al: International physical activity questionnaire: 12-country reliability and validity. Med Sci Sports Exerc 2003, 35(8):1381-1395.

67. Doniger GM, Simon ES, Zivotofsky AZ: Comprehensive computerized assessment of cognitive sequelae of a complete 12-16 hour fast. Behav Neurosci 2006, 120(4):804-816.

68. Doniger GM, Dwolatzky T, Zucker DM, et al: Computerized cognitive testing battery identifies mild cognitive impairment and mild dementia even in the presence of depressive symptoms. Am J Alzheimers Dis Other Demen 2006, 21(1):28-36.

69. Herman T, Mirelman A, Giladi N, Schweiger A, Hausdorff JM: Executive control deficits as a prodrome to falls in healthy older adults: a prospective study linking thinking, walking, and falling. J Gerontol A BiOl Sci Med Sci 2010, 65(10):1086-92.

70. Mirelman A, Herman T, Brozgol M, et al: Executive function and falls in older adults: new findings from a five-year prospective study link fall risk to cognition. PLoS One 2012, 7(6):e40297.

71. Hoops S, Nazem S, Siderowf AD, et al: Validity of the MoCA and MMSE in the detection of $\mathrm{MCl}$ and dementia in Parkinson disease. Neurology 2009, 73(21):1738-1745
72. Nasreddine ZS, Phillips NA, Bedirian V, et al: The Montreal Cognitive Assessment, MoCA: a brief screening tool for mild cognitive impairment. J Am Geriatr Soc 2005, 53(4):695-699.

73. Fals-Stewart W: An interrater reliability study of the Trail Making Test (Parts A and B). Percept Mot Skills 1992, 74(1):438-450.

74. Sanchez-Cubillo I, Perianez JA, drover-Roig D, et al: Construct validity of the Trail Making Test: role of task-switching, working memory, inhibition/ interference control, and visuomotor abilities. J Int Neuropsychol Soc 2009, 15(3):438-450

75. Cantin JF, McFadyen BJ, Doyon J, Swaine B, Dumas D, Vallee M: Can measures of cognitive function predict locomotor behaviour in complex environments following a traumatic brain injury? Brain Inj 2007, 21(3):327-334.

76. Lezak MD: Neuropsychological assessment. Oxford: Oxford press; 2012

77. Riazi A, Hobart JC, Lamping DL, et al: Using the SF-36 measure to compare the health impact of multiple sclerosis and Parkinson's disease with normal population health profiles. J Neurol Neurosurg Psychiatry 2003, 74(6):710-714.

78. Weinberger $M$, Nagle B, Hanlon JT, et al: Assessing health-related quality of life in elderly outpatients: telephone versus face-to-face administration. J Am Geriatr Soc 1994, 42(12):1295-1299.

79. Hauer K, Yardley L, Beyer N, et al: Validation of the falls efficacy scale and falls efficacy scale international in geriatric patients with and without cognitive impairment: results of self-report and interview-based questionnaires. Gerontology 2010, 56(2):190-199.

80. Hauer KA, Kempen GI, Schwenk M, et al: Validity and sensitivity to change of the falls efficacy scales international to assess fear of falling in older adults with and without cognitive impairment. Gerontology 2011, 57(5):462-472

81. Hilbe JM: Negative Binominal Regression. 2nd edition. Cambridge, UK: Cambridge University Press; 2011

82. Panel on Prevention of Falls in Older Persons AGS/BGS: Summary of the Updated American Geriatrics Society/British Geriatrics Society Clinical Practice Guideline for Prevention of Falls in Older Persons. J Am Geriatr Soc 2011, 59(1):148-57.

83. Medical Research Council: A framework for development and evaluation of RCT's for complex interventions to improve health 2000. Internet communication: http://www.mrc.ac.uk/Utilities/Documentrecord/index. htm?d=MRC003372.

84. Faes MC, Reelick MF, Esselink RA, Rikkert MG: Developing and evaluating complex healthcare interventions in geriatrics: the use of the medical research council framework exemplified on a complex fall prevention intervention. J Am Geriatr Soc 2010, 58(11):2212-2221.

85. Faes MC, Reelick MF, Joosten-Weyn Banningh LW, Gier M, Esselink RA, Olde Rikkert MG: Qualitative study on the impact of falling in frail older persons and family caregivers: foundations for an intervention to prevent falls. Aging Ment Health 2010, 14(7):834-842.

86. Reelick MF, Faes MC, Esselink RA, Kessels RP, Olde Rikkert MG: How to perform a preplanned process evaluation for complex interventions in geriatric medicine: exemplified with the process evaluation of a complex falls-prevention program for community-dwelling frail older fallers. J Am Med Dir Assoc 2011, 12(5):331-336.

87. Schwenk M, Lauenroth A, Stock C, et al: Definitions and methods of measuring and reporting on injurious falls in randomised controlled fall prevention trials: a systematic review. BMC Med Res Methodol 2012, 12:50.

doi:10.1186/1471-2377-13-15

Cite this article as: Mirelman et al:: V-TIME: a treadmill training program augmented by virtual reality to decrease fall risk in older adults: study design of a randomized controlled trial. BMC Neurology 2013 13:15. 